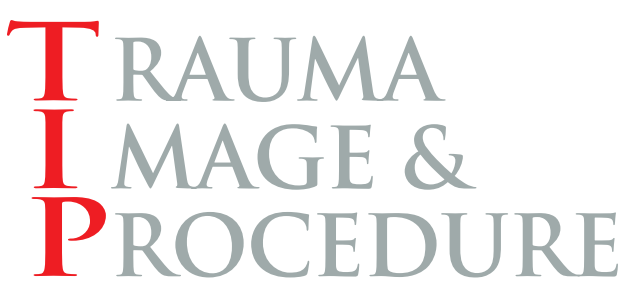

Brief Image in Trauma

Trauma Image Proced $2021 ; 6(1): 5-8$

https://doi.org/10.24184/tip.2021.6.1.5

elSSN 2508-8033 • pISSN 2508-5298

\title{
Unpredictable cardiac arrest caused by blunt cardiac injuries with sternum fracture: a case report
}

\author{
Miyoung Jang ${ }^{1}$, Pil Young Jung ${ }^{2}$ \\ ${ }^{1}$ Department of Surgery, Kangwon National University Hospital, Chuncheon, ${ }^{2}$ Department of \\ Surgery, Yonsei University Wonju College of Medicine, Wonju Severance Christian Hospital, \\ Trauma Center, Wonju, Korea
}

Received September 3, 2021

Revised October 15, 2021

Accepted October 18, 2021

Correspondence to

Pil Young Jung

Department of Surgery, Yonsei

University Wonju College of Medicine,

Wonju Severance Christian Hospital,

Trauma Center, 20 Ilsan-ro, Wonju

26426, Korea

Tel: +82-33-741-0882

Fax: +82-33-741-0574

E-mail: surgery4trauma@yonsei.ac.kr
Blunt cardiac injury (BCI) is common in road traffic accidents due to high energy injuries to the thorax and its structures, such as the anterior and posterior of the chest wall. The severity of $\mathrm{BCI}$ is high enough to cause many on-site deaths. However, patients with $\mathrm{BCI}$ may also be asymptomatic and exhibit normal findings; thus, diagnosis can be significantly challenging for many clinicians. Moreover, since BCI can be diagnosed through multiple serial tests, it is very important to always consider the possibility of BCI in high-risk patients . Diagnosis of BCI begins with suspicion of BCI, followed by repeated electrocardiograms, echocardiography, and cardiac biomarkers measurements along with intensive observation.

Key Words: Blunt cardiac injury, Heart injuries, Myocardial contusions, Thoracic injuries

\section{Introduction}

Blunt cardiac injury (BCI) with blunt chest trauma is mainly caused during motor vehicle accidents, and $20 \%$ of deaths after such accidents are related to BCI $(1,2)$. BCI occurs when the heart is strongly pressed between the sternum and spine by a strong force with an anterior and posterior vector component of the chest wall. Therefore, $\mathrm{BCI}$ is usually accompanied by damage to structures adjacent to the heart, such as ribs, sternum, lung, and spine; however, these injuries are not considered essential for the diagnosis of BCI (3). BCI can be diagnosed with serial checks of clinical features, electrocardiograms (ECG), echocardiography, and cardiac biomarkers measurements, such as creatine kinase (CK), creatine kinase-MB (CK$\mathrm{MB}$ ), and troponin-I (4). Consequently, it is difficult to diagnose BCI without any suspicion or indication of this blunt chest trauma. This report highlights a case of a patient with BCI whose ECG and cardiac biomarkers measurements revealed abnormalities, but echocardiography exhibited normal findings.

\section{Case presentation}

A 64-year-old healthy female patient was presented at our institution. The patient stated that she had collided head-on with a power pole and the steering wheel hit her chest strongly. At the time of admission, the patient was

(c)This is an Open Access article distributed under the terms of the Creative Commons Attribution Non-Commercial License (http://creativecommons.org/licenses/by-nc/4.0) which permits unrestricted noncommercial use, distribution, and reproduction in any medium, provided the original work is properly cited.

Copyright (c) 2021 Korean Association for Research, Procedures and Education on Trauma. All rights reserved. 

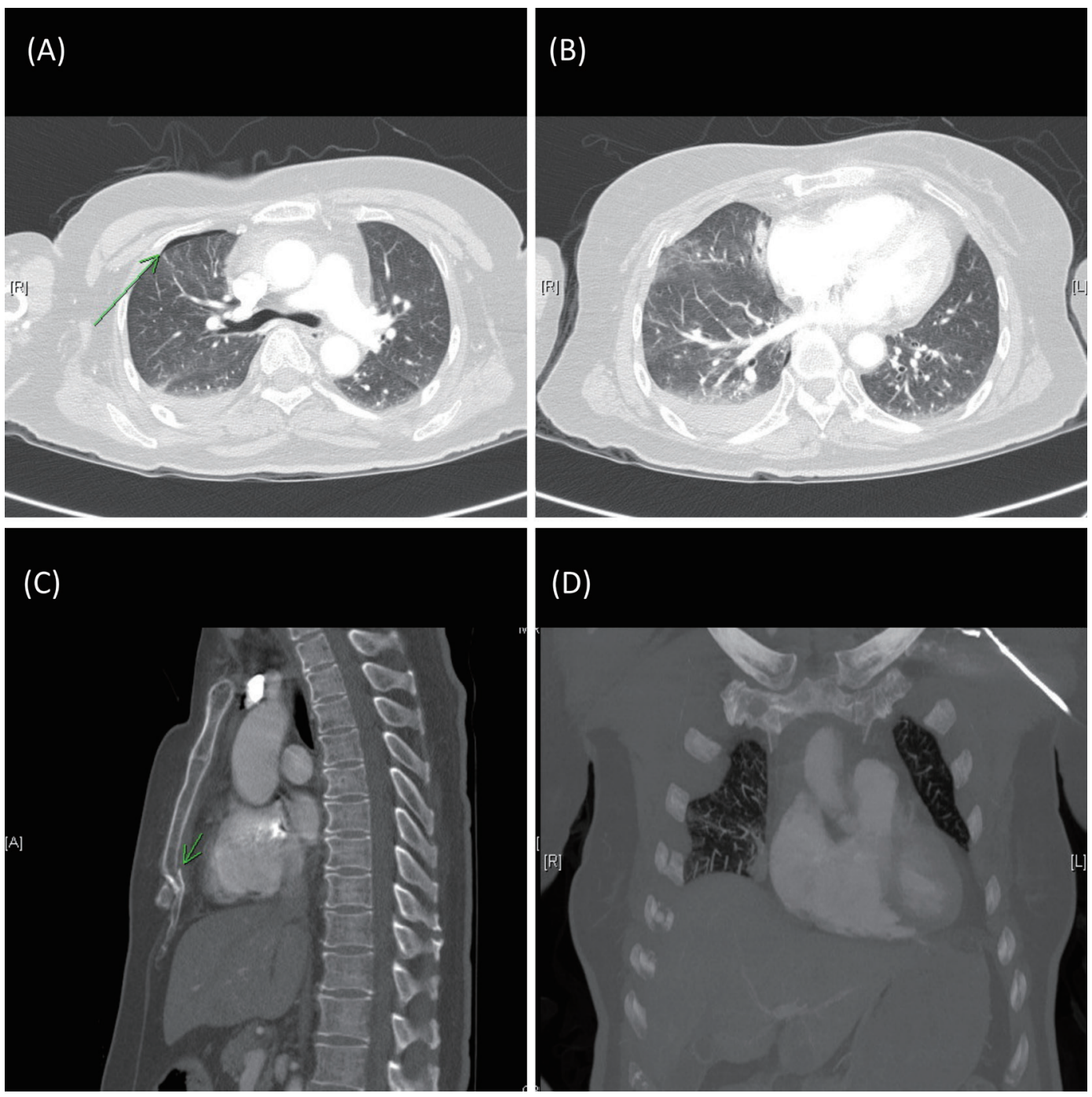

Fig. 1. Chest computed tomography of the patient. (A) Pneumothorax (green arrow), (B) hemothorax, (C) fracture of sternum (green arrow), and (D) multiple rib fracture.

alert and complained of chest wall pain. Initial blood pressure (BP) was $85 / 44 \mathrm{mmHg}$, and heart rate(HR) was 106 beats/min. After infusion of $1 \mathrm{~L}$ of normal saline for 30 mins, BP elevated to $116 / 74 \mathrm{mmHg}$ and $\mathrm{HR}$ descended to 85 beats/min. As the patient's vital signs were improving, computed tomography (CT) was performed. Right pneumothorax and hemothorax with multiple rib and sternum fractures were identified by the chest CT 40 mins after admission (Fig. 1). Closed thoracostomy to the right chest was performed at $1 \mathrm{~h}$ postadmission and approximately $200 \mathrm{~mL}$ of bloody pleural fluid was drained. Consequently, it was decided that the patient should be admitted to the intensive care unit (ICU) for close observation.

Initial laboratory findings revealed that hemoglobin was
$13.2 \mathrm{~g} / \mathrm{dL}$ (normal range; $12.0-16.0 \mathrm{~g} / \mathrm{dL}$ ), CK was $289 \mathrm{U} / \mathrm{L}$, CK-MB was $10.02 \mathrm{ng} / \mathrm{mL}$, and troponin-I was $2.25 \mathrm{ng} / \mathrm{mL}$. A normal sinus rhythm in ECG monitor was observed.

Two hours after arrival, the patient was still in the emergency room because the ICU was not ready. ST-elevation was observed on the ECG monitor. Immediately, a 12-lead ECG test was performed, and ST-elevation with reciprocal change was detected in leads II, III, and aVF (Fig. 2). The emergency medicine specialist performed echocardiography, which exhibited normal cardiac function and features. Although the ECG monitor did not return to normal after 30 mins, the patient's vital signs remained stable.

Echocardiography was performed at $3 \mathrm{~h}$ postadmission. Patient's vital signs remained stable, and she was admit- 


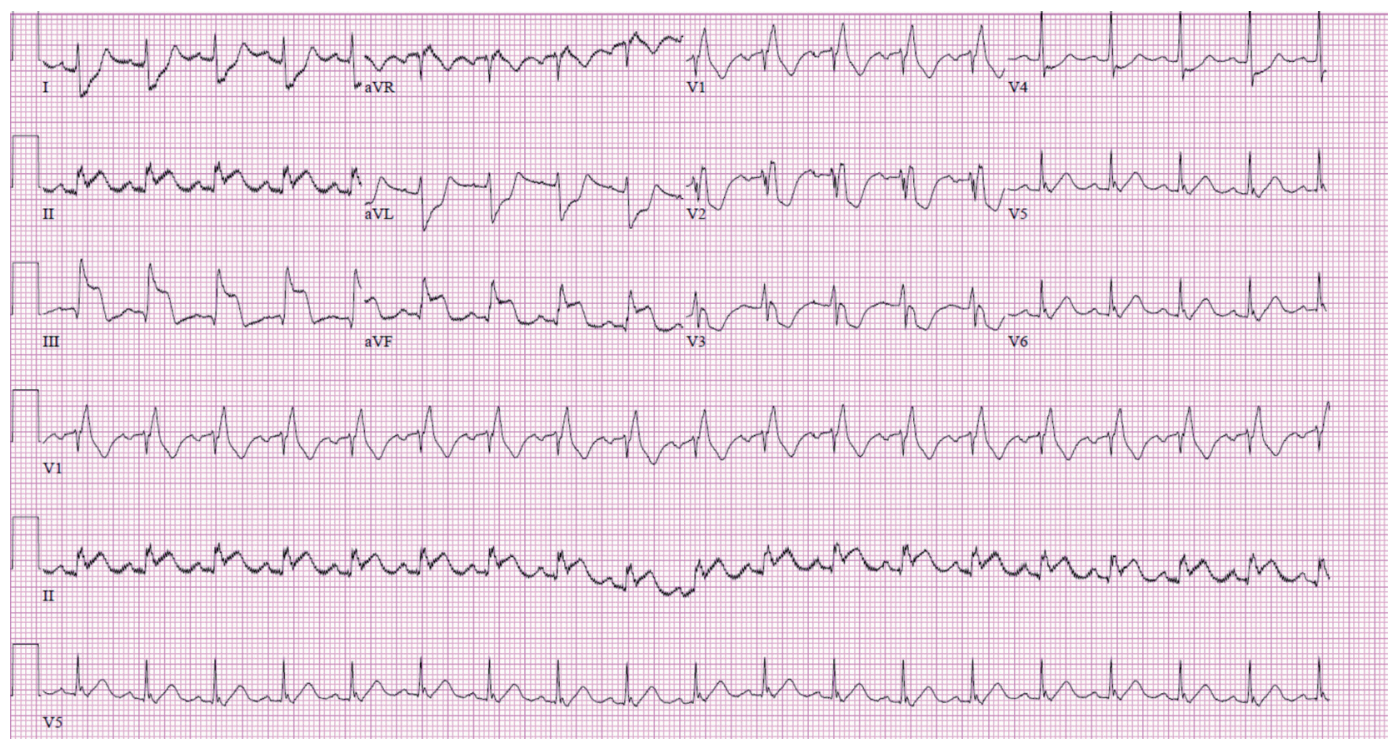

Fig. 2. Patient's electrocardiogram.

ted to the ICU. However, the patient looked pale, and her pulse was not palpated upon arrival at ICU. After 2 mins of cardiopulmonary resuscitation (CPR), the patient had return of spontaneous circulation (ROSC), and her BP and $\mathrm{HR}$ were $55 / 25 \mathrm{mmHg}$ and 42 beats/min, respectively. Intubation was immediately performed; however, ventricular tachycardia rhythm was assessed on the ECG monitor. Despite defibrillation and 50 mins of CPR, the patient could not have ROSC. Application of extracorporeal membrane oxygenation was not performed because of the already prolonged total CPR time. Finally, the patient died $4 \mathrm{~h}$ postadmission.

\section{Discussion}

The incidence of traumatic blunt cardiac injuries varies from $8 \%-86 \%$ (5). Because of its high mortality rate, it is important to diagnose BCI early to implement appropriate treatment. However, BCI cannot be diagnosed with a single test, and can only be suspected through repeated tests $(2,4)$. Because less severe BCIs have nonspecific ECG and cardiac biomarkers abnormalities (6), the possibility of developing $\mathrm{BCI}$ should always be considered when detecting injuries of structures near the heart. When BCI is suspected, the patient must be immediately admitted to the ICU for intensive observation, and prepared for an unpredictable situation $(1,2,4,7)$. ST-elevation myocardial infarction (STEMI) occurs in $12 \%$ of BCI, and urgent coronary angi- ography is essential when detecting STEMI $(2,7)$.

Several serious errors can be found in this report pertaining to BCI treatment. First, the patient had no underlying diseases, but considering her age and injuries of the structures around the heart, BCI should have been suspected since the beginning. Second, no additional tests (e.g., serial ECG or echocardiography or cardiac marker follow-up) were performed. Third, admission to the ICU was significantly delayed despite the need for intensive observations. Fourth, although ST-elevation was observed on ECG, coronary angiography was not performed.

In conclusion, clinicians should always consider the possibility of BCI when treating patients with motor vehicle accidents accompanied by blunt chest trauma, and thus always strive for the early diagnosis and treatment of BCI and its respective complications.

\section{Conflict of interest}

No potential conflict of interest relevant to this article was reported.

\section{References}

1. Marcolini EG, Keegan J. Blunt cardiac injury. Emerg Med Clin North Am. 2015;33(3):519-27.

2. Singh S, Heard M, Pester JM, Angus LD. Blunt Cardi- 
ac Injury. Florida: StatPearls; 2021.

3. Yousef R, Carr JA. Blunt cardiac trauma: a review of the current knowledge and management. Ann Thorac Surg. 2014;98(3):1134-40.

4. El-Menyar A, Al Thani H, Zarour A, Latifi R. Understanding traumatic blunt cardiac injury. Ann Card Anaesth. 2012;15(4):287-95.

5. Fadel R, El-Menyar A, ElKafrawy S, Gad MG. Trau- matic blunt cardiac injuries: An updated narrative review. Int J Crit Illn Inj Sci. 2019;9(3):113-9.

6. Kaye P, O’Sullivan I. Myocardial contusion: emergency investigation and diagnosis. Emerg Med J. 2002;19(1):8-10.

7. Kara H, Avci A, Akinci M, Degirmenci S, Bayir A, Ak A. Blunt chest trauma as a cause of acute myocardial infarction. Acta Clin Belg. 2014;69(5):367-70. 